\title{
Investigation of the idiosyncratic hepatotoxicity of Polygonum multiflorum Thunb. through metabolomics using GC-MS
}

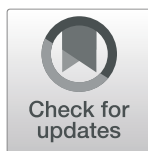

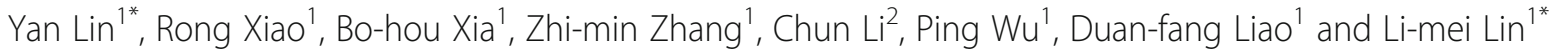

\begin{abstract}
Background: The idiosyncratic hepatotoxicity of Polygonum multiflorum (PM) has attracted considerable interest, but the idiosyncratically hepatotoxic components and endogenous metabolite changes resulting from idiosyncratic hepatotoxicity of PM are not well understood. The aim of this study was to identify the idiosyncratically hepatotoxic components and potential endogenous metabolic biomarkers for PM-induced liver injury.

Methods: Serum biochemical indicators and hematoxylin and eosin (H\&E) staining were evaluated to identify pathological changes. Gas chromatography/mass spectrometry (GC-MS) was performed to identify changes in metabolic biomarkers. Orthogonal projection to latent structures discriminant analysis (OPLS-DA) was applied to determine group clustering trends and differential metabolites.

Results: The results for the liver index, the liver function index and liver pathology showed that Polygonum multiflorum ethanol extract (PME), 50\% ethanol elution fractions and tetrahydroxystilbene glucoside (TSG) from PME can induce idiosyncratic hepatotoxicity. TSG was the main idiosyncratically hepatotoxic component. Forty endogenous metabolites were identified in the rat liver. Six biomarkers, including lower levels of L-valine and higher levels of 3-hydroxybutyric acid, hexadecanoic acid, ribose, phosphoric acid and oxalic acid, were related to PM-induced liver injury. These differential biomarkers led to disruptions in amino acid, fatty acid, oxalate, energy and glucose metabolism. A total of 32 types of endogenous metabolites were identified in rat serum. Ten biomarkers were related to the liver injury induced by TSG, including lower levels of L-valine and L-proline and higher levels of urea, caproic acid, DL-malic acid, D-mannose, 3-hydroxybutyric acid, D-galactose, octadecane and hexadecanoic acid. These differential biomarkers led to disruptions in amino acid, glucose and fat metabolism. The mechanism of idiosyncratic hepatotoxicity in PM involves TSG-induced disruptions in amino acid metabolism, lipid metabolism, energy metabolism and glucose metabolism.
\end{abstract}

Conclusions: These findings reflect the material basis and metabolic mechanism of idiosyncratic PM hepatotoxicity. Keywords: Polygonum multiflorum Thunb., Idiosyncratically hepatotoxic components, Tetrahydroxystilbene glucoside, Mechanism of idiosyncratic hepatotoxicity, Differential biomarkers

\footnotetext{
*Correspondence: linyan198210@163.com; lizasmile@163.com

1 College of Pharmacy, Hunan University of Chinese Medicine, Changsha 410208, China

Full list of author information is available at the end of the article
}

(C) The Author(s). 2021 Open Access This article is licensed under a Creative Commons Attribution 4.0 International License, which permits use, sharing, adaptation, distribution and reproduction in any medium or format, as long as you give appropriate credit to the original author(s) and the source, provide a link to the Creative Commons licence, and indicate if changes were made. The images or other third party material in this article are included in the article's Creative Commons licence, unless indicated otherwise in a credit line to the material. If material is not included in the article's Creative Commons licence and your intended use is not permitted by statutory regulation or exceeds the permitted use, you will need to obtain permission directly from the copyright holder. To view a copy of this licence, visit http://creativecommons.org/licenses/by/4.0/ The Creative Commons Public Domain Dedication waiver (http://creativecommons.org/publicdomain/zero/1.0/) applies to the data made available in this article, unless otherwise stated in a credit line to the data. 


\section{Background}

Polygonum multiflorum Thumb. (PM) is widely used in traditional Chinese medicine and as a dietary supplement, but hepatotoxicity due to PM occurs in certain individuals. The biological effects of PM include detoxification, carbuncle elimination, bowel relaxation, malaria prevention, antiaging and hair blackening [1]. Although PMinduced liver injuries have increased significantly, some investigations of suspected clinical patients have revealed that such injuries occur in only a minority of patients and are related to idiosyncratic hepatotoxicity [2-5]. The hepatotoxicity of PM has also been summarized and recorded in the LiverTox ${ }^{\odot}$ database, a comprehensive resource for idiosyncratic drug-induced liver injury (IDILI) produced by the National Institute of Diabetes and Digestive and Kidney Diseases and National Library of Medicine [4]. Although IDILI often occurs in a minority of patients (generally $<1 \%$ ) [6], it is one of the leading causes of drug development failure and drug withdrawal from the market. IDILIs are often found after marketing or in the final phase of a clinical study since IDILIs cannot be evaluated in preclinical drug safety assessments using healthy animals [7]. Thus, animal models must be developed to assess drugs that cause IDILI. The inflammatory stress hypothesis has provided some of the first animal models of idiosyncratic hepatotoxicity in which nontoxic doses of IDILI-causing drugs are rendered hepatotoxic upon coexposure to a nontoxic but modestly inflammatory dose of bacterial endotoxin (lipopolysaccharide (LPS)) [8, 9]. The LPS model has been successfully used to evaluate several drugs known to cause IDILI in humans, including trovafloxacin, ranitidine, sulindac, chlorpromazine, halothane, monocrotaline, amiodarone and diclofenac [6]. In addition, combined treatment with a nontoxic dose of LPS and a therapeutic dose of PM resulted in acute idiosyncratic liver injury in rats [2]. In this study, the LPS model is used to evaluate the idiosyncratic hepatotoxicity of PM.

The components of PM include tetrahydroxystilbene glucoside (TSG), anthraquinone, phospholipids and tannins [10]. TSG, anthraquinone, tannins and biotoxins may be hepatotoxic components of PM [10,11], but no definitive conclusion has been reached. Thus, the relationship between the hepatotoxic components and toxicity to the liver must be elucidated. Some scholars have reported the toxic effects of PM, but few have elucidated the components and metabolic mechanism underlying idiosyncratic hepatotoxicity. In this study, liver injuries in rats treated with Polygonum multiflorum ethanol extract (PME) and the effects of different elution fractions and TSG under nonidiosyncratic and idiosyncratic models were systematically investigated. The purpose of this study was to identify idiosyncratically hepatotoxic components and elucidate the metabolic mechanism of idiosyncratic hepatotoxicity due to PM. Gas chromatography/mass spectrometry (GC-MS)-based metabolomics was adopted to characterize PM-induced idiosyncratic hepatotoxicity and to explore the underlying mechanism. Unlike other functional genomic tools, metabolomics can provide a detailed and specific profile of the endogenous metabolic status of an organism in response to toxicological events. GC-MS is highly advantageous for detecting low-molecular-weight metabolites in metabonomic studies because GC-MS provides heightened equipment stability and user-friendly tools for data analysis. Untargeted metabolomics methods have been used to simultaneously detect several classes within the metabolome, allowing observation of changes in endogenous metabolites that are linked to toxicity. This study will provide an experimental basis for the primary hepatotoxic components in PM and elucidate the metabolic mechanism of hepatotoxicity.

\section{Methods \\ Plant material}

PM pieces were provided by the Hubei Yafei TCM Company (Batch number: 20170809) and were confirmed to be the dry root of Polygonum multiflorum by Limin Gong, an associate professor of the School of Pharmacy of Hunan University of Chinese Medicine.

\section{Materials and reagents}

Chlorpromazine hydrochloride was obtained from Harvest Pharmaceutical Co., Ltd. (Shanghai, China). An alanine transaminase (ALT) kit and an aspartate transaminase (AST) kit were purchased from Nanjing Jiancheng Bioengineering Institute (Nanjing, China). A lactate dehydrogenase (LDH) kit was obtained from Wuhan Huamei Biotech Co., Ltd., China. Methoxyamine, N, Obis (trimethylsilyl) trifluoroacetamide, pyridine, malic acid and LPS were purchased from Sigma Chemical Company (St. Louis, USA).

\section{Drug preparation \\ PME}

A certain quantity of the PM powder $(16 \mathrm{~kg})$ was weighed, and three extractions of $2 \mathrm{~h}$ each were accomplished using eightfold, sixfold and sixfold dilutions of $70 \%$ ethanol. The extracts were combined and concentrated to dryness under a vacuum.

Different elution fractions of PME: portions of the PME were applied to macroporous resin and sequentially eluted with water, 50\% ethanol and 95\% ethanol. Each elution fraction was concentrated to dryness.

\section{TSG}

TSG was prepared in our laboratory. The structure was confirmed by comparing the compound's ${ }^{1} \mathrm{H}$ and ${ }^{13} \mathrm{C}$ 
NMR spectra to reported spectra. Examination by HPLC showed that the purity reached $98 \%$ [12].

\begin{abstract}
Animals
Specific-pathogen-free (SPF) male rats $(200 \pm 20 \mathrm{~g})$ were purchased from Hunan SJA Laboratory Animal Co., Ltd.; male rats are more susceptible to drug-induced idiosyncratic hepatotoxicity [5]. The animal permit number is SCXK (Xiang) 2016-0002. The temperature and humidity of the animal housing conditions met the requirements for housing experimental animals. The rats were kept under a 12-h dark-light cycle and were housed for one week prior to the experiments. Animal care and treatments were conducted according to established guidelines and protocols approved by Animal Care and Use Committee of Hunan University of Chinese Medicine (Changsha, China). All efforts were made to minimize the number of animals used and their suffering.
\end{abstract}

\section{Animal grouping and pharmacological intervention}

According to the Chinese Pharmacopoeia, 3-6g of raw PM is suggested for human clinical application. The corresponding clinical dose for a rat was determined to be $0.3125-0.625 \mathrm{~g} / \mathrm{kg}$. We selected the dose of PM according to this requirement, the actual administration volume and references [2]. We selected the dosages of the water elution fraction, $50 \%$ ethanol elution fraction and 95\% ethanol elution fraction according to the yields of the different elution fractions of PM. The yields of the water elution fraction, 50\% ethanol elution fraction and $95 \%$ ethanol elution fraction were 10,20 and $10 \%$, respectively. In addition, we selected the dose of TSG according to the percentage TSG content in PM (1\%). We selected only one dose for the water fraction according to preexperiment results, which showed that cotreatment with a nontoxic dose of LPS and water fraction $(5.4 \mathrm{mg} / \mathrm{kg})$ did not induce idiosyncratic hepatotoxicity.

A total of 240 male rats were randomly divided into 30 groups ( $n=8$ per group) assigned to the following treatments: the negative control (C); LPS model (M); chlorpromazine-positive group $(\mathrm{P}$, chlorpromazine hydrochloride can cause hepatotoxicity through an inflammatory response $[13,14], 10.4 \mathrm{mg} / \mathrm{kg}$ ); high-, medium- and low-dose PME (CPH 5.4, CPM 1.08 and CPL $0.54 \mathrm{~g} / \mathrm{kg}$, respectively); water elution fraction of PME (W $5.4 \mathrm{mg} /$ $\mathrm{kg}$ ); high-, medium- and low-dose $50 \%$ ethanol elution fractions of PME (FH 1.08, FM 0.216 and FL $0.108 \mathrm{~g} / \mathrm{kg}$, respectively); high-, medium- and low-dose $95 \%$ ethanol elution fractions of PME (NH 54, NM 10.8 and NL 5.4 $\mathrm{mg} / \mathrm{kg}$, respectively); high-, medium- and low-dose TSG (TSGH 108, TSGM 10.8 and TSGL $2.7 \mathrm{mg} / \mathrm{kg}$, respectively); LPS + chlorpromazine-positive (PL); LPS + high-, medium- and low-dose PME (CPHL, CPML and CPLL, respectively); LPS + water elution fraction of PME (WL);
LPS + high-, medium- and low-dose 50\% ethanol elution fractions of PME (FHL, FML and FLL, respectively); LPS + high-, medium- and low-dose $95 \%$ ethanol elution fractions of PME (NHL, NML and NLL, respectively); and LPS + TSG high-, medium- and low-dose groups (TSGH L, TSGML and TSGLL, respectively). The groupings and acronyms for each group are shown in Table 1 . The $\mathrm{C}$ and $\mathrm{M}$ groups were given normal saline by oral gavage each day, and the other groups were given $5-\mathrm{mL} / \mathrm{kg}$ aliquots by oral gavage. The M group and LPS + drug groups were injected with $2.8 \mathrm{mg} / \mathrm{kg}$ LPS at $4 \mathrm{~h}$ after drug administration each day [15]. Pharmacological intervention was carried out for 15 consecutive days. Livers were collected and weighed. Serum samples were prepared by centrifuging the collected blood samples (at $2000 \mathrm{rpm}$ for $10 \mathrm{~min}$ at $4{ }^{\circ} \mathrm{C}$ ) and then stored at $-80^{\circ} \mathrm{C}$. At the end of the treatment, rats were weighed and injected with a high concentration of pentobarbital sodium $(80 \mathrm{mg} / \mathrm{kg})$ for anesthesia, blood was collected from the abdominal aorta, and cervical dislocation was performed.

\section{Liver index and biochemical index assays}

The liver index values were calculated using Eq. 1. The activities of ALT, AST and LDH were measured using the corresponding kits with a microplate reader (Thermo Electron Corporation, USA).

Liver index values $=($ liver weight $/$ rat weight $) * 100 \%$

\section{Hematoxylin and eosin (H\&E) staining of idiosyncratic hepatotoxicity}

The hepatic tissue samples were fixed in $10 \%$ formalin. Then, the paraffin-embedded tissue samples were sectioned into $5-\mu \mathrm{m}$-thick slices. The sections were stained with $H \& E$ and examined under a light microscope (Model IX71, Olympus, Tokyo, Japan).

\section{GC-MS-based hepatic and serum metabolomics study GC-MS specimen pretreatment [16]}

Liver homogenate/serum samples $(100 \mu \mathrm{L})$ were thawed at $4{ }^{\circ} \mathrm{C}$ for $1 \mathrm{~h}$ and mixed with $50 \mu \mathrm{L}$ of internal standard $(1 \mathrm{mg} / \mathrm{mL}$ malic acid) by vortexing. After vortex mixing with $450 \mu \mathrm{L}$ of methanol, each sample was allowed to settle for $8 \mathrm{~min}$ and then centrifuged at 13,000 RPM for $10 \mathrm{~min}$. The supernatant was aspirated and dried under nitrogen. Methoxyamine pyridine $(50 \mu \mathrm{L}, 20 \mathrm{mg} / \mathrm{mL})$ was added to the dried pellet, and the mixture was incubated for $1 \mathrm{~h}$ at $70^{\circ} \mathrm{C}$. Next, $100 \mu \mathrm{L}$ of N, O-bis (trimethylsilyl) trifluoroacetamide was added to the solution, mixed for $30 \mathrm{~s}$ and then incubated at $70^{\circ} \mathrm{C}$ for $1 \mathrm{~h}$ and at room temperature for $2 \mathrm{~h}$. The sample was centrifuged at $13000 \mathrm{RPM}$ for $8 \mathrm{~min}$, and then $100 \mu \mathrm{L}$ of 
Table 1 The groupings and acronyms for each group

\begin{tabular}{|c|c|c|c|}
\hline Groupings & Acronyms & Groupings & Acronyms \\
\hline $\begin{array}{l}\text { negative control } \\
(2.8 \mathrm{mg} / \mathrm{kg})\end{array}$ & C & $\begin{array}{l}\text { LPS model } \\
(2.8 \mathrm{mg} / \mathrm{kg})\end{array}$ & $M$ \\
\hline $\begin{array}{l}\text { chlorpromazine-positive } \\
\text { (10.4 mg/kg) }\end{array}$ & P & $\begin{array}{l}\text { LPS + chlorpromazine-positive } \\
(2.8 \mathrm{mg} / \mathrm{kg}+10.4 \mathrm{mg} / \mathrm{kg})\end{array}$ & $P L$ \\
\hline $\begin{array}{l}\text { high low dose PME } \\
(5.4 \mathrm{~g} / \mathrm{kg})\end{array}$ & $\mathrm{CPH}$ & $\begin{array}{l}\text { LPS + high dose PME } \\
(2.8 \mathrm{mg} / \mathrm{kg}+5.4 \mathrm{mg} / \mathrm{kg})\end{array}$ & $\mathrm{CPHL}$ \\
\hline $\begin{array}{l}\text { medium dose PME } \\
(1.08 \mathrm{~g} / \mathrm{kg})\end{array}$ & CPM & $\begin{array}{l}\text { LPS + medium dose PME } \\
(2.8 \mathrm{mg} / \mathrm{kg}+1.08 \mathrm{~g} / \mathrm{kg})\end{array}$ & CPML \\
\hline $\begin{array}{l}\text { Low dose PME } \\
(0.54 \mathrm{~g} / \mathrm{kg})\end{array}$ & $\mathrm{CPL}$ & $\begin{array}{l}\text { LPS + low dose PME } \\
(2.8 \mathrm{mg} / \mathrm{kg}+0.54 \mathrm{~g} / \mathrm{kg})\end{array}$ & CPLL \\
\hline $\begin{array}{l}\text { water elution fraction of PME } \\
(5.4 \mathrm{mg} / \mathrm{kg})\end{array}$ & W & $\begin{array}{l}\text { LPS + water elution fraction of PME } \\
(2.8 \mathrm{mg} / \mathrm{kg}+5.4 \mathrm{mg} / \mathrm{kg})\end{array}$ & $W L$ \\
\hline $\begin{array}{l}\text { high dose } 50 \% \text { ethanol elution fraction of PME } \\
(1.08 \mathrm{~g} / \mathrm{kg})\end{array}$ & $\mathrm{FH}$ & $\begin{array}{l}\text { LPS + high dose } 50 \% \text { ethanol elution fraction of PME } \\
(2.8 \mathrm{mg} / \mathrm{kg}+1.08 \mathrm{~g} / \mathrm{kg})\end{array}$ & $\mathrm{FHL}$ \\
\hline $\begin{array}{l}\text { medium dose } 50 \% \text { ethanol elution fraction of PME } \\
(0.216 \mathrm{~g} / \mathrm{kg})\end{array}$ & FM & $\begin{array}{l}\text { LPS + medium dose } 50 \% \text { ethanol elution fraction of PME } \\
(2.8 \mathrm{mg} / \mathrm{kg}+0.216 \mathrm{~g} / \mathrm{kg})\end{array}$ & FML \\
\hline $\begin{array}{l}\text { low dose } 50 \% \text { ethanol elution fraction of PME } \\
(0.108 \mathrm{~g} / \mathrm{kg})\end{array}$ & $\mathrm{FL}$ & $\begin{array}{l}\text { LPS + low dose } 50 \% \text { ethanol elution fraction of PME } \\
(2.8 \mathrm{mg} / \mathrm{kg}+0.108 \mathrm{~g} / \mathrm{kg})\end{array}$ & FLL \\
\hline $\begin{array}{l}\text { high low dose TSG of PME } \\
(108 \mathrm{mg} / \mathrm{kg})\end{array}$ & TSGH & $\begin{array}{l}\text { LPS + high dose TSG of PME } \\
(2.8 \mathrm{mg} / \mathrm{kg}+108 \mathrm{mg} / \mathrm{kg})\end{array}$ & TSGHL \\
\hline $\begin{array}{l}\text { medium dose TSG of PME } \\
(10.8 \mathrm{mg} / \mathrm{kg})\end{array}$ & TSGM & $\begin{array}{l}\text { LPS + medium dose TSG of PME } \\
(2.8 \mathrm{mg} / \mathrm{kg}+10.8 \mathrm{mg} / \mathrm{kg})\end{array}$ & TSGML \\
\hline $\begin{array}{l}\text { low dose TSG of PME } \\
(2.7 \mathrm{mg} / \mathrm{kg})\end{array}$ & TSGL & $\begin{array}{l}\text { LPS + low dose TSG of PME } \\
(2.8 \mathrm{mg} / \mathrm{kg}+2.7 \mathrm{mg} / \mathrm{kg})\end{array}$ & TSGLL \\
\hline
\end{tabular}

the supernatant was transferred to a $250-\mu \mathrm{L}$ internal tube that was placed into a sample vial for GC-MS analysis using a model QP2010 gas chromatograph-mass spectrometer (QP2010, Shimadzu, Japan).

\section{GC-MS conditions [16]}

A DB-5MS quartz capillary column $(30 \mathrm{~m} \times 0.25 \mathrm{~mm} \times$ $0.25 \mu \mathrm{m}$, Agilent J\&W Scientific, Folsom, USA) was used. The injector temperature was set to $280{ }^{\circ} \mathrm{C}$, the injection volume was $1 \mu \mathrm{L}$, and the split ratio was $1: 1$. The flow rate of helium (carrier gas, 99.999\%) was set to 1.0 $\mathrm{mL} / \mathrm{min}$. The column temperature was initially $70^{\circ} \mathrm{C}$, and this temperature was held for $4 \mathrm{~min}$, gradually increased at a rate of $20^{\circ} \mathrm{C} / \mathrm{min}$ to $110^{\circ} \mathrm{C}$ and then at a rate of $8^{\circ} \mathrm{C} / \mathrm{min}$ to $270{ }^{\circ} \mathrm{C}$ and then held for $5 \mathrm{~min}$. The temperature of the electron ionization source was $200{ }^{\circ} \mathrm{C}$. The solvent delay duration was $6.5 \mathrm{~min}$, and the MS scanning range was $35-550 \mathrm{~m} / \mathrm{z}$. During the analysis, the sample injection sequence was randomly arranged, and one quality control (QC) sample was added every nine samples.

\section{GC-MS data processing and analysis}

Chromatograms were subjected to ion-pair extraction, peak alignment, peak matching and peak amplitude correction using XCMS software. A data set of the samples consisting of retention times and peak areas of metabolites was imported into SIMCA-P software (version 14.0,
Umetrics AB, Umeå, Sweden) for orthogonal projections to latent structures discriminant analysis (OPLS-DA). Variables with importance parameter values (variable influence on projection, VIP) exceeding 1 in the OPLS-DA model were selected as potential differential metabolites. SPSS 13.0 statistical software (SPSS Inc., Chicago, USA) was used to perform $t$-tests. Metabolites with VIP $>1$ and $P<0.05$ ( $t$-test) were considered statistically significant. Biomarkers were determined by comparison with biological databases, including the Kyoto Encyclopedia of Genes and Genomes (KEGG) and the Human Metabolome Database (HMDB).

\section{Statistical analysis}

The data are represented as the mean \pm SD and were analyzed by SPSS 13.0 software. Student's $t$-tests were used for comparisons between groups. One-way analysis of variance (ANOVA) was used for comparisons among groups. Nonparametric data were analyzed by MannWhitney $U$-tests. $P<0.05$ was considered statistically significant.

\section{Results}

Effects on the liver index

As a commonly used toxicology indicator, the liver index reflects liver toxicity. Compared to that in the control group, liver index values were significantly lower in the high-, medium- and low-dose TSG groups. The liver 
index values did not differ significantly in the other groups, and the results indicated that direct administration of PME, different elution fractions and TSG did not induce significant liver injuries (Fig. 1a). Compared with that in the model group, the liver index values in all the other groups except for the water elution fraction increased significantly in the LPS model (Fig. 2a). Direct administration of PME, the 50\% ethanol elution fraction and TSG did not induce liver injury, but PME, the 50\% ethanol elution fraction of PME and TSG administered with LPS caused liver injury. The results showed that the hepatotoxicity of PM was idiosyncratic.

\section{Effects on the liver function index}

One of the key markers of liver damage is the release of enzymes such as AST, ALT and LDH into the circulatory system. As shown in Fig. 1b-d, compared to those in the $\mathrm{C}$ group, the levels of AST were significantly higher in the $\mathrm{P}, \mathrm{CPH}, \mathrm{FH}$ and TSGH groups $(P<0.01)$ and in the NH group $(P<0.05)$; the levels of ALT were significantly higher in the $\mathrm{P}, \mathrm{CPH}, \mathrm{CPM}, \mathrm{FH}$ and $\mathrm{NH}$ groups $(\mathrm{P}<0.01)$ and in the $\mathrm{CPL}, \mathrm{NM}$ and $\mathrm{FM}$ groups $(\mathrm{P}<0.05)$; and the levels of LDH were also significantly elevated in the P, CPH, CPM, CPL, FH, FM, NH, TSGH and TSGM groups $(P<0.01)$ and in the FL group $(\mathrm{P}<$ 0.05). As shown in Fig. 2b-d, compared to those in the $M$ group, the levels of ALT, AST and LDH increased significantly in the PL, CPHL, CPML, CPLL, FHL, FHM, TSGHL, TSGML and TSGLL groups $(\mathrm{P}<0.01)$. When the doses of TSG and PM increased, the levels of ALT, AST and LDH increased. The results indicated that TSG was the main idiosyncratically hepatotoxic component of PM, and the idiosyncratic hepatotoxicity of TSG was dose dependent. The levels of ALT were higher in the PME groups than in the TSG groups, and the results indicate that the other components may induce hepatotoxicity.

\section{Liver pathology examination}

Direct administration of PME, different elution fractions of PME and TSG did not induce any significant pathological changes (Supplementary Fig. 1). The livers of the rats treated with LPS alone showed moderate infiltration of inflammatory cells. Severe liver injury was observed in the rats cotreated with LPS and the high dose of PME. The liver injuries included widespread hepatocyte necrosis, the disappearance of nuclei, moderate interstitial fibrosis and some infiltrated inflammatory cells. The nuclei of the hepatocytes were misshapen in the rats cotreated with LPS and the high-dose 50\% ethanol elution fractions of PME. Hepatocyte necrosis was observed in the rats cotreated with LPS and the high dose of TSG (Fig. 3).
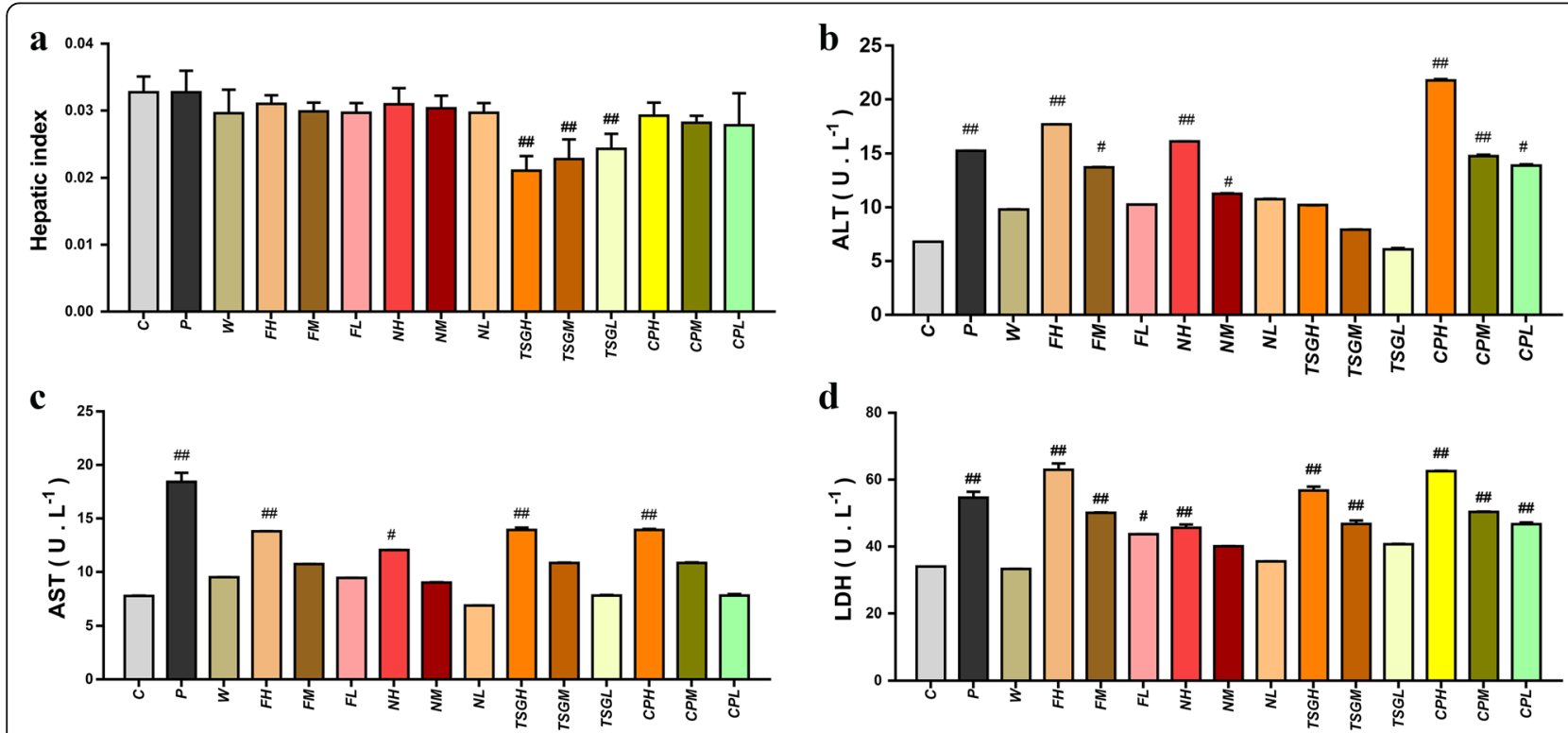

Fig. 1 The effects of Polygonum multiflorum ethanol extract (PME), different fractions of PME and tetrahydroxystilbene glucoside (TSG) on the liver index and serum AST, ALT and LDH under nonidiosyncratic models. a Liver index. $\mathbf{b}$ The level of AST. c The level of ALT. $\mathbf{d}$ The level of LDH (D). \#\#: $P<0.01$ compared to the $C$ group, $\#$ : $P<0.05$ compared to the $C$ group; Negative control group (C); Chlorpromazine-positive group (P); high, medium and low doses of PME (CPH, CPM and CPL, respectively); water elution fraction of PME (W); high, medium and low doses of the 50\% ethanol elution fraction of PME (FH, FM and FL, respectively); high, medium and low doses of the $95 \%$ ethanol elution fraction of PME (NH, NM and $\mathrm{NL}$, respectively); and high, medium and low doses of TSG (TSGH, TSGM and TSGL, respectively) 


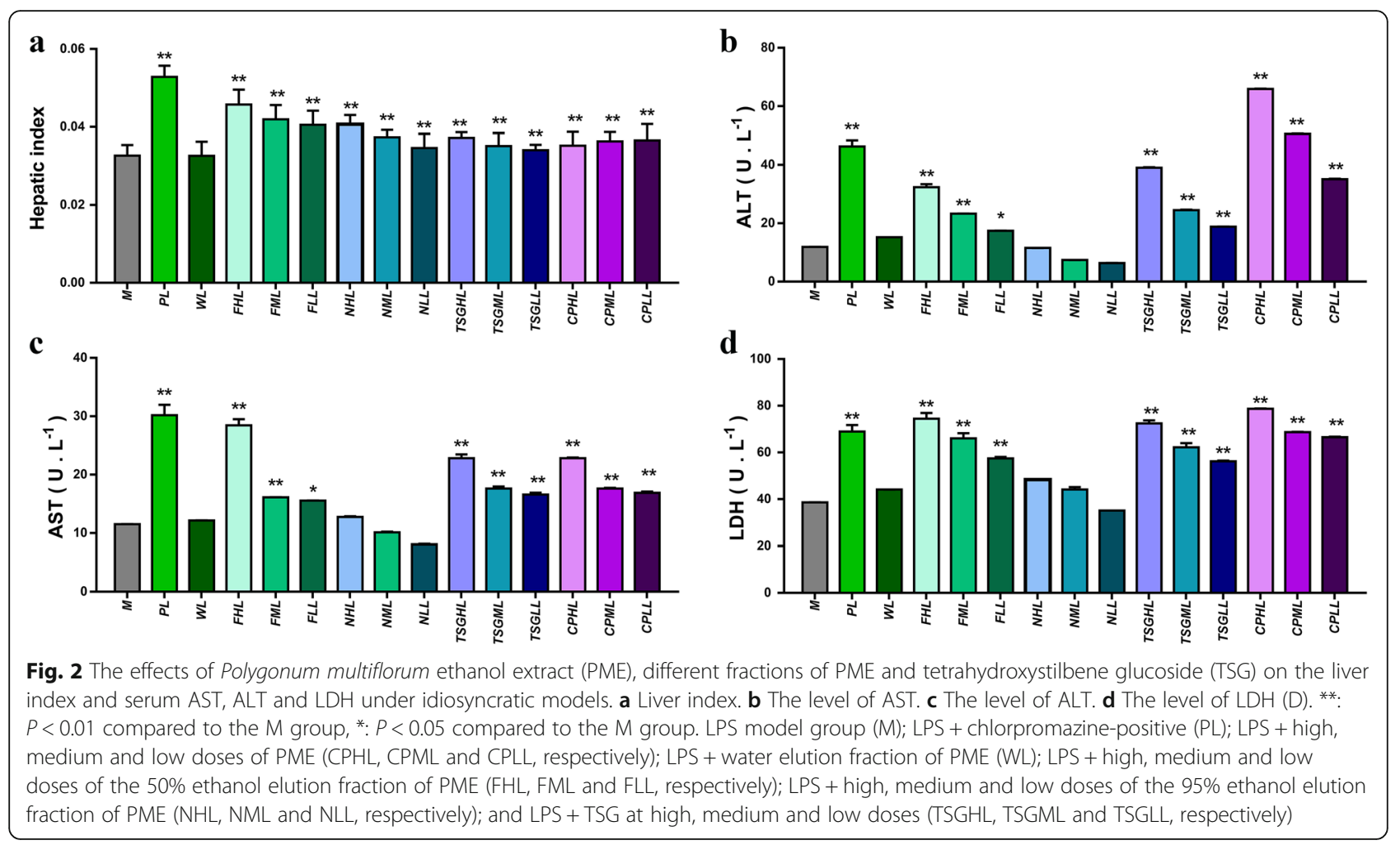

\section{GC-MS-based hepatic metabolomics study}

To clarify the mechanism underlying idiosyncratic PM hepatotoxicity, the metabolites showing differences between the negative control group and the LPS model group were identified and analyzed, and the differential metabolites between the M group and the CPHL groups were identified and analyzed. A total of 40 types of endogenous metabolites, including amino acids, carbohydrates, oxalate and fatty acids, were identified. The results are shown in Table 2 and Fig. 4a. The explanatory ability (given by the parameter $\mathrm{R}_{2} \mathrm{Y}$ ) and the predictive ability (given by the parameter $\mathrm{Q}_{2}$ ) of the model



Fig. 3 Comparison of the severity of rat liver injury (H\&E staining, 200x, hepatic histological changes (arrow)). Negative control group (C); LPS model group (M); LPS + chlorpromazine-positive (PL); LPS + high dose of PME (CPHL); LPS + high dose of the $50 \%$ ethanol elution fraction of PME (FHL); LPS + high dose of the 95\% ethanol elution fraction of PME (NHL); and LPS + TSG at high doses (TSGHL) 
Table 2 Hepatic differential metabolites and metabolic pathways in the different groups

\begin{tabular}{|c|c|c|c|c|c|c|}
\hline No & $\mathrm{t} / \mathrm{min}$ & Metabolites & $\mathrm{m} / \mathbf{z}$ & Trend & & Metabolic pathways \\
\hline 1 & 7.014 & Propanoic acid & 74.08 & & & \\
\hline 2 & 7.417 & I-Valine & 117.15 & & & \\
\hline 3 & 7.673 & I-Alanine & 89.09 & $\downarrow^{1)}$ & $\downarrow^{2)}$ & Amino acid metabolism \\
\hline 4 & 7.988 & Glycine & 75.07 & $\uparrow^{1)}$ & & Amino acid metabolism \\
\hline 5 & 8.267 & Ethanedioic acid & 90.03 & & $\uparrow^{2)}$ & Oxalate metabolism \\
\hline 6 & 8.518 & 3-Hydroxybutyric acid & 104.10 & & $\uparrow^{2)}$ & Energetic metabolism \\
\hline 7 & 8.577 & leucine & 265.30 & & & \\
\hline 8 & 8.894 & I-Isoleucine & 131.17 & & & \\
\hline 9 & 9.964 & Urea & 60.06 & & & \\
\hline 10 & 10.133 & Serine & 105.09 & & & \\
\hline 11 & 10.277 & Silanamine & 80.59 & & & \\
\hline 12 & 10.386 & Silanol & 90.20 & & & \\
\hline 13 & 10.535 & Hexadecane & 260.65 & & & \\
\hline 14 & 10.732 & L-Threonine & 119.12 & $\uparrow^{1)}$ & & Amino acid metabolism \\
\hline 15 & 10.982 & Glycine & 75.07 & & & \\
\hline 16 & 11.143 & Butanedioic acid & 118.09 & & & \\
\hline 17 & 11.424 & Pyrimidine & 80.09 & & & \\
\hline 18 & 11.667 & 2-Butenedioic acid & 116.07 & & & \\
\hline 19 & 11.763 & Serine & 105.09 & & & \\
\hline 20 & 12.168 & $\mathrm{~N}, \mathrm{O}, \mathrm{O}$-Tris (trimethylsilyl)-L-threonine & 257.40 & & & \\
\hline 21 & 12.846 & I-Aspartic acid, & 133.10 & & & \\
\hline 22 & 13.541 & Aminomalonic acid & 119.08 & & & \\
\hline 23 & 13.851 & Malic acid & 134.09 & & & \\
\hline 24 & 13.968 & Fructose benzoyl oxime & 176.17 & & & \\
\hline 25 & 14.342 & L-Aspartic acid & 133.10 & & & \\
\hline 26 & 14.416 & L-Proline & 115.13 & & & \\
\hline 27 & 14.576 & 2-Pyrrolidone-5-carboxylic acid & 151.10 & & & \\
\hline 28 & 15.892 & Glutamine & 169.11 & & & \\
\hline 29 & 16.675 & D-Ribose & 150.13 & & $\uparrow^{2)}$ & Glucose metabolism \\
\hline 30 & 17.933 & Phosphoric acid & 98.00 & & $\uparrow^{2)}$ & Amino acid metabolism \\
\hline 31 & 19.62 & D-Mannose & 182.17 & $\uparrow^{1)}$ & & Glucose metabolism \\
\hline 32 & 19.845 & D-Glucose & 180.16 & $\uparrow^{1)}$ & & Glucose metabolism \\
\hline 33 & 20.062 & D-Galactose & 180.16 & & & \\
\hline 34 & 21.887 & Hexadecanoic acid & 256.42 & & & \\
\hline 35 & 22.29 & Inositol & 180.16 & & & \\
\hline 36 & 22.76 & D-Mannitol & 182.17 & & & \\
\hline 37 & 22.837 & Octadecane & 254.49 & & & \\
\hline 38 & 23.898 & Oleic acid & 282.46 & & & \\
\hline 39 & 24.268 & Octadecanoic acid & 284.48 & & & \\
\hline 40 & 28.251 & Hexadecanoic acid & 256.42 & & $\uparrow^{2)}$ & Fatty acid metabolism \\
\hline
\end{tabular}

Notes: LPS model group (M) vs. negative control group, ${ }^{1)} p<0.01$, lipopolysaccharide (LPS) + PM high dose group (CPHL) vs. LPS model group ${ }^{2)} p<0.01$.

$\uparrow$-increase, $\downarrow$-decrease. Negative control group (C) 


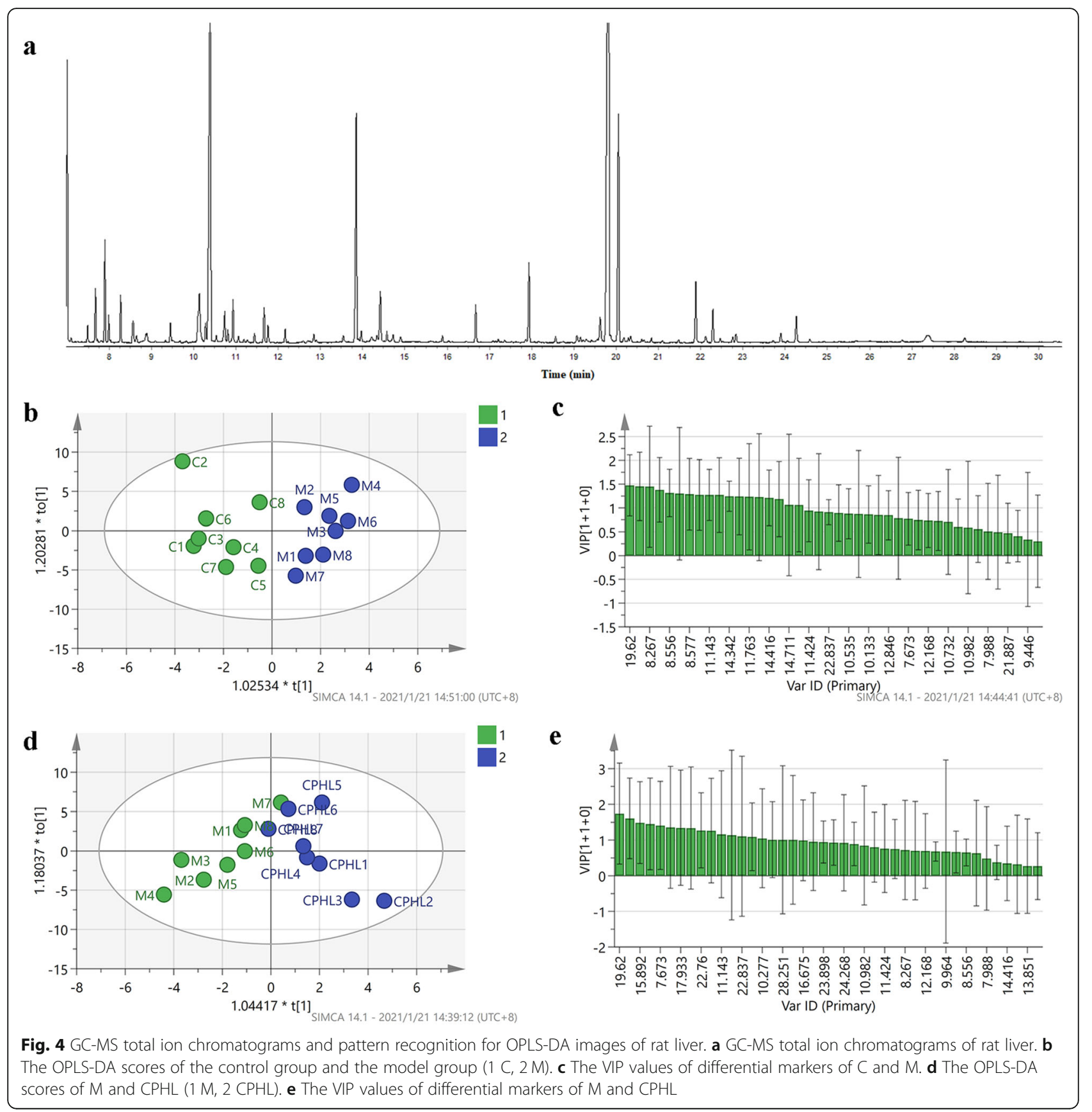

established for the $C$ and $M$ groups were 0.836 and 0.463 , respectively. The $\mathrm{R}_{2} \mathrm{Y}$ and $\mathrm{Q}_{2}$ of the model for the $\mathrm{M}$ and CPHL groups were 0.844 and 0.632 , respectively. These results indicated that these models showed high degrees of differentiation and prediction.

As shown in Fig. 4b-c, the hepatic metabolites in the $M$ group were significantly different from those in the $C$ group. The hepatic metabolites in the $\mathrm{M}$ group were significantly different from those in the CPHL groups. The differential metabolites were screened based on VIP values from the OPLS-DA model (Fig. 4d-e). Variables with VIP values $>1$ were considered critical for classification. The screened differential metabolites were subjected to significance tests, and variables with $\mathrm{P}$ values < 0.05 were considered to be associated with the idiosyncratic model and idiosyncratic hepatotoxicity. Compared to the $\mathrm{C}$ group, five variables involving glucose metabolism and amino acid metabolism were different in the $\mathrm{M}$ group, namely, reduced levels of L-valine, L-threonine, mannose and glucose and an elevated alanine level. Compared to the M group, the CPHL group showed one decreased variable (L-valine) and five increased variables, 
namely, 3-hydroxybutyric acid, hexadecanoic acid, ribose, phosphoric acid and oxalic acid. These hepatic metabolite changes showed that PM led to disruptions in the metabolism of amino acids, lipids, oxalate, energy and glucose. These results suggest that the mechanism of PM idiosyncratic hepatotoxicity involves disruption of amino acid, lipid, oxalate, energy and glucose metabolism.

\section{GC-MS-based serum metabolomics study}

The results for the liver index, liver function index and liver pathology suggested that hepatotoxicity was idiosyncratic for high-dose PME, the 50\% ethanol elution fractions of PME and TSG. Therefore, in this study, differential metabolites were identified between the control group and the LPS model group. High-dose PME, the $50 \%$ ethanol elution fraction of PME and TSG were able to induce serious idiosyncratic hepatotoxicity; thus, the differential metabolites were identified between the $M$ group and the CPHL, FHL and TSGH groups.

A total of 32 types of endogenous metabolites were identified, including amino acids, carbohydrates and fatty acids. The results are shown in Table 3 and Fig. 5a. The explanatory ability (given by the parameter $\mathrm{R}_{2} \mathrm{Y}$ ) and the predictive ability (given by the parameter $\mathrm{Q}_{2}$ ) of the model established for the $\mathrm{C}$ and $\mathrm{M}$ groups were 0.880 and 0.574 , respectively. The $R_{2} Y$ and $Q_{2}$ values of the model for the M and CPHL groups were 0.966 and 0.894, respectively. The $\mathrm{R}_{2} \mathrm{Y}$ and $\mathrm{Q}_{2}$ of the model for the $\mathrm{M}$ and FHL groups were 0.788 and 0.561 , respectively. The $R_{2} Y$ and $Q_{2}$ of the model for the $M$ and TSGHL groups were 0.789 and 0.532 , respectively. These results indicate that these models featured high degrees of differentiation and prediction.

As shown in Fig. 5 b1-e1, the serum metabolites in the $\mathrm{M}$ group were significantly different from those in the $\mathrm{C}$ group. Compared to those in the $\mathrm{M}$ group, the serum metabolites in the CPHL, FHL and TSGHL groups were significantly different. The differential metabolites were screened based on the VIP values of the OPLS-DA model (Fig. 5b2-e2). Variables with VIP values $>1$ were considered to play critical roles in classification. The screened differential metabolites were subjected to significance tests, and variables with $P$ values $<0.05$ were thought to be associated with the idiosyncratic model and idiosyncratic hepatotoxicity. Compared to those in the $\mathrm{C}$ group, eight variables involving glucose metabolism and amino acid metabolism in the $\mathrm{M}$ group were altered, namely, reduced levels of L-valine, L-leucine, Lthreonine, L-proline, citric acid, malic acid and Dgalactose and an increased level of alanine. Compared to the $M$ group, the CPHL group showed two decreased variables (L-valine and L-leucine) and seven increased variables, namely, the levels of D-mannose, D-galactose,
D-glucose, 3-hydroxybutyric acid, urea, caproic acid and hexadecanoic acid. The FHL group showed nine differential variables, namely, lower levels of L-valine and Lthreonine and increased levels of glycine, 3hydroxybutyric acid, urea, D-mannose, fructose, Dgalactose oleic acid and hexadecanoic acid. The TSGHL group showed 10 differential variables, namely, lower levels of L-valine and L-proline and higher levels of urea, caproic acid, DL-malic acid, D-mannose, 3hydroxybutyric acid, D-galactose, octadecane and hexadecanoic acid. The serum metabolite changes showed that PME, the 50\% ethanol elution fraction of PME and TSG all led to disruptions in amino acid metabolism, lipid metabolism, energy metabolism and glucose metabolism in the idiosyncratic model. In addition, TSG was the main chemical component of PME and the 50\% ethanol elution fraction. These results suggest that the mechanism underlying idiosyncratic PME hepatotoxicity was that TSG led to disruptions in amino acid metabolism, lipid metabolism, energy metabolism and glucose metabolism.

\section{Discussion}

PM is a traditional Chinese medicine and has been used in clinical practice and in the food industry for many years worldwide. However, the idiosyncratic hepatotoxicity of PM has attracted considerable interest. The 50\% ethanol extract of PM $(1.08 \mathrm{~g} / \mathrm{kg})$ may result in liver injury in the idiosyncratic model [2]. In this study, the results for the liver index, liver function index and liver pathology showed that the hepatotoxicity of PM was idiosyncratic. This result may explain why PM at clinically recommended safe doses and in treatment courses is not toxic to most individuals but is toxic to some individuals. TSG has two conformations, the cis and trans forms [15]. Furthermore, another study showed that trans-TSG can be photoisomerized to cis-TSG [17]. Cisstilbene glucoside can induce immunological idiosyncratic hepatotoxicity [3]. Trans-TSG can increase the potential risk of liver injury from cis-TSG. In this study, a small portion of TSG may have been converted to cisTSG during drug administration. This result is consistent with a previous report indicating that the toxicity of PM may be related to the content of tetrahydroxystilbene glucosides [12].

In general, the toxicity induced by a medication is proportional to the dose and the duration of exposure to the drug. Therefore, when the dose of TSG and PM increased, the levels of ALT, AST and LDH increased under nonidiosyncratic models in this study. This intrinsic hepatotoxicity mechanism was related to drug accumulation. Given that most people taking PM products at the recommended therapeutic dose do not develop liver injury, the intake of direct toxic components may not be 
Table 3 Serum differential metabolites and metabolic pathways in the diffirent groups

\begin{tabular}{|c|c|c|c|c|c|c|c|c|}
\hline \multirow[t]{2}{*}{ No } & \multirow[t]{2}{*}{$t / \min$} & \multirow[t]{2}{*}{ Metabolites } & \multirow[t]{2}{*}{$\mathrm{m} / \mathrm{z}$} & \multicolumn{4}{|c|}{ Trend } & \multirow[t]{2}{*}{ Metabolic pathways } \\
\hline & & & & $M$ & CPHL & FHL & TSGHL & \\
\hline 1 & 6.544 & 4-Methylvaleric acid & 116.16 & & & & & \\
\hline 2 & 6.924 & Propanoic acid & 74.00 & & & & & \\
\hline 3 & 7.414 & L-Valine & 117.15 & $\downarrow^{1)}$ & $\downarrow^{2)}$ & $\downarrow^{2)}$ & $\downarrow^{2)}$ & Amino acid metabolism \\
\hline 4 & 7.811 & Alanine & 89.09 & $\uparrow^{1)}$ & & & & Amino acid metabolism \\
\hline 5 & 8.214 & Glycine & 75.07 & & & $\uparrow^{2)}$ & & Amino acid metabolism \\
\hline 6 & 8.518 & 3-Hydroxybutyric acid & 104.11 & & $\uparrow^{2)}$ & $\uparrow^{2)}$ & $\uparrow^{2)}$ & Energetic metabolism \\
\hline 7 & 8.818 & L-Leucine & 116.16 & $\downarrow^{1)}$ & $\downarrow^{2)}$ & & & Amino acid metabolism \\
\hline 8 & 9.444 & Methoxyacetlc acid & 89.07 & & & & & \\
\hline 9 & 9.964 & Urea & 60.06 & & $\uparrow^{2)}$ & $\uparrow^{2)}$ & $\uparrow^{2)}$ & Microbial metabolism \\
\hline 10 & 10.09 & L-Serine & 105.09 & & & & & \\
\hline 11 & 10.67 & L-Threonine & 119.09 & $\downarrow^{1)}$ & & & & Amino acid metabolism \\
\hline 12 & 10.88 & 3-Aminoisobutyric acid & 103.12 & & & & & \\
\hline 13 & 12.124 & Hexadecanoic acid & 116.16 & & $\uparrow^{2)}$ & & $\uparrow^{2)}$ & Glucose metabolism \\
\hline 14 & 12.198 & Methoxyacetic acid & 90.08 & & & & & \\
\hline 15 & 13.514 & DL-Methionine & 131.17 & & & & & \\
\hline 16 & 13.809 & DL-Malic acid & 134.09 & & & & & \\
\hline 17 & 14.364 & L-Proline & 115.13 & $\downarrow^{1)}$ & & & $\downarrow^{2)}$ & Amino acid metabolism \\
\hline 18 & 16.648 & Ribitol & 152.15 & & & & & \\
\hline 19 & 17.155 & Sulfurous & 82.08 & & & & & \\
\hline 20 & 18.709 & Citric Acid & 192.12 & $\downarrow^{1)}$ & & $\uparrow^{2)}$ & $\uparrow^{2)}$ & Glucose metabolism \\
\hline 21 & 19.608 & D-Mannose & 180.16 & & $\uparrow^{2)}$ & $\uparrow^{2)}$ & $\uparrow^{2)}$ & Glucose metabolism \\
\hline 22 & 19.759 & D-Glucose & 180.16 & & $\uparrow^{2)}$ & $\uparrow^{2)}$ & & Carbohydrate metabolism \\
\hline 23 & 20.026 & D-Galactose & 180.16 & $\downarrow^{1)}$ & $\uparrow^{2)}$ & $\uparrow^{2)}$ & $\uparrow^{2)}$ & Glucosemetabolism \\
\hline 24 & 21.826 & Palmitic Acid & 256.42 & & & & & \\
\hline 25 & 22.27 & Inositol & 180.16 & & & & & \\
\hline 26 & 22.802 & Hexadecane & 224.43 & & & & & \\
\hline 27 & 23.845 & Octadecane & 254.49 & & $\uparrow^{2)}$ & & $\uparrow^{2)}$ & Fatty acid biosynthesis \\
\hline 28 & 23.92 & Oleic acid & 282.46 & & & $\uparrow^{2)}$ & & Amino acid metabolism \\
\hline 29 & 24.215 & Octadecanoic acid & 284.48 & & & & & \\
\hline 30 & 25.33 & Cholesterol & 386.65 & & & & & \\
\hline 31 & 25.629 & Arachidonic acid & 304.46 & & & & & \\
\hline 32 & 28.174 & Hexadecanoic acid & 256.42 & & & $\uparrow^{2)}$ & & Amino acid metabolism \\
\hline
\end{tabular}

Notes: LPS model group (M) vs. Negative control group, ${ }^{1)} \mathrm{p}<0.01$, Lipopolysaccharide (LPS) + PM high dose group (CPHL), LPS + $50 \%$ ethanol elution fraction high dose group (FHL), LPS + TSG high dose groups (TSGHL) vs. LPS model group respectively, ${ }^{2)} p<0.01$. $\uparrow$-Increase, $\downarrow$-Decrease

sufficient to damage the liver but instead only initiates very mild hepatic cell stress, which can be resisted by the tissue regeneration, repair or antistress systems that maintain the normal structure and functions of hepatocytes exposed to low concentrations of toxic components [18]. In this study, direct administration of PME, different elution fractions of PME and TSG did not induce any significant pathological changes, which is consistent with the previous study [18]. Cotreatment with LPS at a nontoxic dose and PM at the clinically equivalent dose causes obvious liver injury in a dose- dependent manner. When the body is in a state of immunological activation, components (e.g., trans-TSG) with immunoenhancing activity might increase an individual's susceptibility to potential toxic components (e.g., cis-TSG), leading to extensive injury of hepatic cells and overexpression of inflammatory cytokines [19]. An allergic constitution in a patient causes the metabolites and components of PM to be treated as haptens. After binding to their macromolecular carriers, the haptens form covalently bound whole antigens, which induce the production of antibodies and hypersensitivity [20]. In 


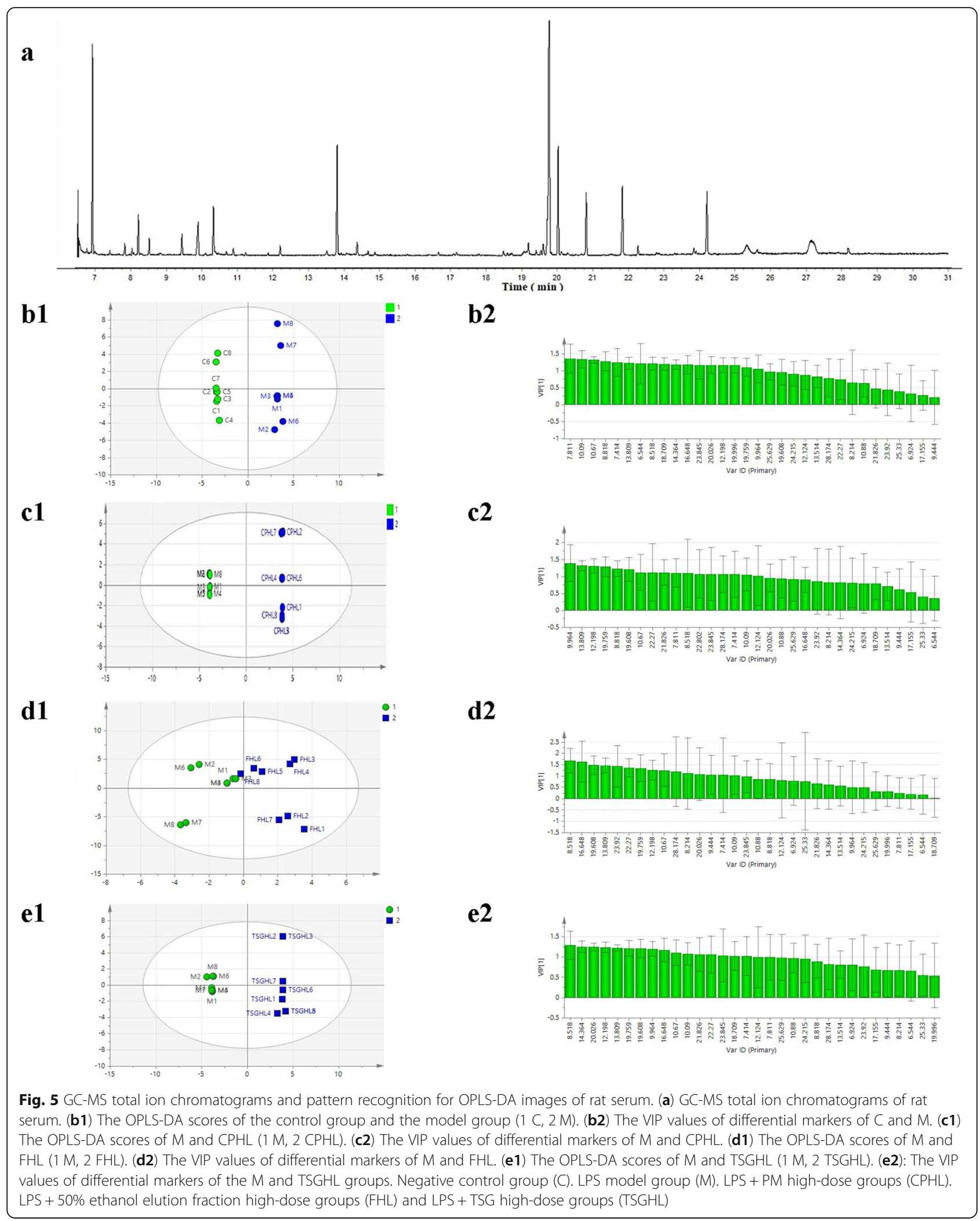


this study, the metabolites and components of PM and TSG may have induced immunological activation, immunoenhancing activity or haptens, which would promote idiosyncratic hepatotoxicity. Therefore, when the dose of TSG and PM increased, the levels of ALT, AST and LDH increased under idiosyncratic models. We think that idiosyncratic PM-induced liver injury is a result of complex interactions between drugs/reactive metabolites and the host immune response. However, the complex interactions between drugs/reactive metabolites and the host immune response must be clarified in further studies.

The high dose of PM $(40 \mathrm{~g} / \mathrm{kg})$ altered lipid metabolism, amino acid metabolism and bile acid metabolism and excretion in a dose-dependent manner related to the mechanism of liver injury [21]. Cotreatment with a nontoxic dose of LPS and ethyl acetate extract resulted in clear liver injury, which mainly involved two pathways: tricarboxylic acid cycle and sphingolipid metabolism [22]. Rats treated with PM exhibited significant disturbances in energy metabolism and amino acid metabolism [23, 24]. In this study, liver injury in the rats treated with PME, different elution fractions and TSG under idiosyncratic models were systematically investigated. GC-MS-based hepatic and serum metabolomics was adopted to characterize PM-induced idiosyncratic hepatotoxicity and to explore the underlying mechanism. This study showed that the mechanism of PM-induced hepatotoxicity was based on disruption of energy metabolism and amino acid metabolism by TSG, explaining the mechanism of PM-induced hepatotoxicity. The liver plays important roles in fatty acid synthesis, $\beta$ oxidation metabolism and the maintenance of fatty acid levels, and 3-hydroxybutyric acid is an intermediate in fatty acid metabolism [25]. The increase in 3hydroxybutyric acid indicated fatty acid metabolism disorder. Increased free fatty acid levels also indicated an abnormal tricarboxylic acid cycle and $\beta$-oxidation, suggesting that hepatotoxicity may be caused by hepatocytic mitochondria injury. The elevated levels of urea suggest that nitrogen-containing metabolites are increased. Nitrogenous wastes produced from protein and amino acid decomposition may induce toxicity.

\section{Conclusions}

TSG was the main idiosyncratic hepatotoxic component, and liver injury increased with increasing TSG doses. A total of 32 types of endogenous metabolites were identified in rat serum. Ten biomarkers were related to the liver injury induced by TSG and led to disruptions in amino acid, glucose and fat metabolism. These findings provide the material basis and metabolic mechanism of PM-induced idiosyncratic hepatotoxicity.

\section{Abbreviations}

PM: Polygonum multiflorum; PME: Polygonum multiflorum ethanol extract: TSG: tetrahydroxystilbene glucoside; LPS: Lipopolysaccharide; ALT: Alanine aminotransferase; AST: Aspartate aminotransferase; LDH: Lactate dehydrogenase; GC-MS: Gas chromatography/mass spectrometry; OPLADA: Orthogonal projection to latent structures discriminant analysis; VIP: Variable influence on projection

\section{Supplementary Information}

The online version contains supplementary material available at https://doi. org/10.1186/s12906-021-03276-4.

Additional file 1: Supplementary Fig. 1. Comparison of the severity of rat liver injury (H\&E staining, 200x). Chlorpromazine-positive group (P); high, medium and low doses of PME (CPH, CPM and $\mathrm{CPL}$, respectively); water elution fraction of PME $(W)$; high, medium and low doses of the $50 \%$ ethanol elution fraction of PME ( $F H, F M$ and $\mathrm{FL}$, respectively); high, medium and low doses of the 95\% ethanol elution fraction of PME $(\mathrm{NH}$, NM and NL, respectively); high, medium and low doses of TSG of PME (TSGH, TSGM and TSGL, respectively); LPS + medium and low doses of PME (CPML and CPLL, respectively), LPS+ water elution fraction of PME $(\mathrm{WL}) ; \mathrm{LPS}+$ medium and low doses of the $50 \%$ ethanol elution fraction of PME (FML and FLL, respectively); LPS + medium and low doses of the 95\% ethanol elution fraction of PME (NML and NLL, respectively); and LPS + TSG at medium and low doses (TSGML and TSGLL, respectively).

\section{Acknowledgements}

Not applicable.

\section{Authors' contributions}

$Y L, L L$ and DF conceived the original idea and designed the study. $Y L, R X$, $\mathrm{ZZ}, \mathrm{CL}$ PW and RX performed the experiments and data analysis. Data interpretation and drafting of the manuscript were performed by $Y L, R X, Z Z$, $C L P W$ and RX. The manuscript was revised by $Y L, L L, D F, B X, Z Z, C L$ and $P W$. All authors reviewed the manuscript and provided final approval for submission.

\section{Funding}

This work was supported by the Key Projects of the National Chinese Medicine and Herb Modernization Foundation, China (2017YFC1701900); the Science foundation of Hunan Province, China (2020JJ6008); the Science Research Projects of the Hunan Provincial Department of Science (Kq1907139) and the Science Research Projects of the Hunan Provincial Department of Education (20C1433); First-class Discipline Project on Chinese Pharmacology of Hunan University of Chinese Medicine (2018-3); the Science Research Projects of Hunan University of Chinese Medicine (No.19). Limei Lin conceived and helped design the study. Yan Lin conceived the original idea, helped design the study, performed the experiments and analyzed the data.

\section{Availability of data and materials}

All of the data analyzed in this study are included in this article.

\section{Declarations}

Ethics approval and consent to participate

All animal treatments and experiments were approved by the Animal Care and Use Committee of the Hunan University of Chinese Medicine, Changsha, China (SCXK (Xiang) 2016-0002).

Consent for publication

Not applicable.

Competing interests

All the authors declare that they have no competing interests. 


\section{Author details}

${ }^{1}$ College of Pharmacy, Hunan University of Chinese Medicine, Changsha 410208, China. ${ }^{2}$ China Institute of Chinese Materia Medica, China Academy of Chinese Medical Sciences, Beijing 100700, China.

Received: 29 September 2020 Accepted: 15 March 2021

Published online: 12 April 2021

\section{References}

1. Liu Y, Wang Q, Yang JB, Guo XH, Liu WX, Ma SC, et al. Polygonum multiflorum thunb.: a review on chemical analysis, processing mechanism, quality evaluation, and hepatotoxicity. Front Pharmacol. 2018;9:e364.

2. Li CY, Li XF, Tu C, Na L, Xiao XH. The idiosyncratic hepatotoxicity of Polygonum multiflorum based on endotoxin model. Acta Pharm Sin B. 2015:50:28-33

3. Meng YK, Li CY, Li RY, He LZ, Cui HR, Yin P, Zhang CE, Li PY, Sang XX, Wang Y, Niu M, Zhang YM, Guo YM, Sun R, Wang JB, Bai ZF, Xiao XH. Cis-stilbene glucoside in Polygonum multiflorum induces immunological idiosyncratic hepatotoxicity in LPS-treated rats by suppressing PPAR-gamma. Acta Pharm Sin B. 2017;38(10):1340-52. https://doi.org/10.1038/aps.2017.32.

4. Wang J, Ma Z, Niu M, Zhu Y, Liang Q, Zhao Y, Song J, Bai Z, Zhang Y, Zhang P, Li N, Meng Y, Li Q, Qin L, Teng G, Cao J, Li B, Chen S, Li Y, Zou Z, Zhou H, Xiao $X$. Evidence chain-based causality identification in herb-induced liver injury: exemplification of a well-known liver-restorative herb Polygonum multiflorum. Front Med Prc. 2015;9(4):457-67. https://doi.org/10.1007/s11 684-015-0417-8.

5. Li YC, Niu M, Bai ZF, Zhang C, Zhao YL, LRY, et al. Screening for main components associated with the idiosyncratic hepatotoxicity of a tonic herb, Polygonum multiflorum. Front Med Prc. 2017;11(2):253-65. https://doi. org/10.1007/s11684-017-0508-9.

6. Shaw PJ, Ganey PE, Roth RA. Idiosyncratic drug-induced liver injury and the role of inflammatory stress with an emphasis on an animal model of trovafloxacin hepatotoxicity. Toxicol Sci. 2010;118(1):7-18. https://doi.org/1 0.1093/toxsci/kfq168.

7. Björnsson ES. Drug-induced liver injury: an overview over the most critical compounds. Arch Toxicol. 2015;89(3):327-34. https://doi.org/10.1007/s002 04-015-1456-2.

8. Poulsen KL, Olivero-Verbel J, Beggs KM, Ganey PE, Roth RA. Trovafloxacin enhances lipopolysaccharide-stimulated production of tumor necrosis factor-a by macrophages: role of the DNA damage response. J Pharmacol Exp Ther. 2014;350(1):164-70. https://doi.org/10.1124/jpet.114.214189.

9. Roth RA, Ganey PE. Animal models of idiosyncratic drug-induced liver injury current status. Crit Rev Toxicol. 2011;41(9):723-39. https://doi.org/10.3109/1 0408444.2011 .575765$.

10. Lin L, Lin H, Zhang M, Ni B, Yin X, Qu C, Ni J. A novel method to analyze hepatotoxic components in polygonum multiflorum using ultraperformance liquid chromatography-quadrupole time-of-flight mass spectrometry. J Hazard Mater. 2015;299:249-59. https://doi.org/10.1016/j.jha zmat.2015.06.014.

11. Lin L, Ni B, Lin H, Zhang M, Li X, Yin X, Qu C, Ni J. Traditional usages, botany, phytochemistry, pharmacology and toxicology of Polygonum multiflorum Thunb.: a review. J Ethnopharmacol. 2015;159:158-83. https:// doi.org/10.1016/j.jep.2014.11.009.

12. Xiao R, Lin Y, Lei SM, Zhang Y, Huang J, Xia BH, et al. Analyzing crude/ processed root of Polygonum multiflorum from different habitats by UPLC fingerprint and mode identification methods. China J Chin Mater Med. 2017:42(12):2305-10

13. Morgan K, Martucci N, Kozlowska A, Gamal W, Brzeszczynki F, Treskes P, et al. Chlorpromazine toxicity is associated with disruption of cell membrane integrity and initiation of a pro-inflammatory response in the hepaRG hepatic cell line. Biomed Pharmacother. 2019;111:1408-16. https:/ doi.org/10.1016/j.biopha.2019.01.020.

14. Brzeszczynska J, Morgan K, Brzeszczynski F, Samuel K, Hayes P, Plevris J. Towards understanding the mechanisms of chlorpromazine-induced hepatic toxicity using a human HepaRG-based model. J Hepatol. 2018;68: S141. https://doi.org/10.1016/S0168-8278(18)30498-7.

15. He LZ, Yin P, Meng YK, Tang JF, He TT, Niu M, et al. Immunological synergistic mechanisms of trans-/cis-stilbene glycosides in Heshouwurelated idiosyncratic liver injury. Sci Bull. 2017;62:748-51.

16. Zhang Z, Zhou Y, Lin Y, Li YM, Xia BH, Lin LM, Liao D. GC-MS-based metabolomics research on the anti-hyperlipidaemic activity of Prunella vulgaris L. polysaccharides. Int J Biol Macromol. 2020;159:461-73. https://doi. org/10.1016/.i.jbiomac.2020.05.003.

17. Sun $J L$, Huang $X L$, Wu HQ, Huang F. Determination of content and light stability of cis- and trans-2,3,5,4'-tetrahydroxystilbene-2-O- $\beta$-Dglucoside in Radix Polygoni multiflori by HPLC/DAD/MS. Chin Pharm J. 2009;44:541-4.

18. Rao T, Liu YT, Zeng XC, Li CP, Ou-Yang DS. The hepatotoxicity of Polygonum multiflorum: the emerging role of the immune-mediated liver injury. Acta Pharmacol Sin. 2020;0:1-9.

19. Bai ZF, Meng YK, He LZ, Tang JF, Xiao XH. Immune idiosyncratic liver injury induced by traditional non-toxic traditional Chinese medicine and a hypothesis of its mechanism. Chin Pharmacol J. 2017:52:1105-9.

20. Liu Y, Wang WP, Sun MY, Ma BR, Pang LN, Du YY, et al. Polygonum multiflorum-induced liver injury: clinical characteristics, risk factors, material basis, action mechanism and current challenges. Front Pharmacol. 2019;10: 1467. https://doi.org/10.3389/fphar.2019.01467.

21. Li YX, Gong XH, Liu MC, Peng CP, Peng L, Wang YT. Investigation of liver injury of Polygonum multiflorum Thunb in rats by metabolomics and traditional approaches. Front Pharmacol. 2017;8:791.

22. Li CY, Tu C, Gao D, Wang RL, Zhang HZ, Niu M, et al. Metabolomic study on idiosyncratic liver injury induced by different extracts of Polygonum multiflorum in rats integrated with pattern recognition and enriched pathways analysis. Front Pharmacol. 2016;7:483.

23. Zhang $Y$, Wang $N$, Zhang $M$, Diao $T$, Lin $G$. Metabonomics study on polygonum multiflorum induced liver toxicity in rats by GC-MS. Int J Clin Exp Med. 2015;8(7):10986-92.

24. Ruan LY, Li MY, Xing YX, Hong W, Chen C, Chen JF, et al. Hepatotoxicity and hepatoprotection of Polygonum multiflorum Thund. As two sides of the same biological coin. J Ethnopharmacol. 2019;230:81-94. https://doi. org/10.1016/j.jep.2018.10.032.

25. Jowett M, Quastel JH. Studies in fat metabolism: the formation and breakdown of acetoacetic acid in animal tissues. Biochem J. 1935;29(9): 2181-91. https://doi.org/10.1042/bj0292181.

\section{Publisher's Note}

Springer Nature remains neutral with regard to jurisdictional claims in published maps and institutional affiliations.

Ready to submit your research? Choose BMC and benefit from:

- fast, convenient online submission

- thorough peer review by experienced researchers in your field

- rapid publication on acceptance

- support for research data, including large and complex data types

- gold Open Access which fosters wider collaboration and increased citations

- maximum visibility for your research: over $100 \mathrm{M}$ website views per year

At BMC, research is always in progress.

Learn more biomedcentral.com/submissions 